\title{
Modelling of the Cooling Process on the Runout Table of a Hot Strip Mill - A Parallel Approach
}

\author{
R. Krishna Kumar, S.K.Sinha \\ Department of Electrical Engineering \\ Indian Institute of Science \\ Bangalore - 560012 \\ INDIA
}

\author{
A.K.Lahiri \\ Department of Metallurgy \\ Indian Institute of Science \\ Bangalore - 560012
}

INDIA

\begin{abstract}
This paper deals with the development of a new model for the cooling process on the runout table of hot strip mills. The suitability of different numerical methods for the solution of the proposed model equation from the point of view of accuracy and computation time are studied. Parallel solutions for the model equation are proposed.

Keywords: Modelling, Hotstrip Mill, Parallel Solution, PDEs
\end{abstract}

\section{Introduction}

In a hot strip mill, the strip is cooled by water sprays from top and bottom on the runout table (ROT). In order to get the desired mechanical and metallurgical properties, the rate of cooling and final temperature of the strip have to be maintained within a predefined band by controlling the flow of water.

This paper deals with the development of a mathematical model for the ROT which can be used for real time simulation of the cooling process on the ROT. The chosen model equation takes into account the latent heat of phase transformation and variation of various thermophysical properties of the strip with temperature. For the solution of the proposed model equation the suitability of three numerical methods, namely, the finite difference method, the orthogonal collocation method and the integral profile method are examined from the point of view of accuracy of the solution and computational time. Since the model equation is to be solved in real time, parallel solutions of the model equation are proposed and the performance of the parallel solutions examined.

\section{The Model Equation}

In a hot strip mill, the strip is cooled on the runout table (ROT) by spraying water from top and bottom. Figure 1 shows a typical ROT.

Convection to the sprayed water and radiation to the atmosphere are the prime modes of heat transfer on the ROT. Conduction along the thickness of the strip and conduction to the work rolls also affect the temperature of the strip in the runout section. Steel being allotropic in nature can have different crystalline structures (phases) in the solid state, each structure being stable within a particular temperature range. The strip undergoes an exothermic phase transformation as it is cooled and the latent heat of phase transformation has an effect on the final temperature of the strip. The latent heat varies with the rate of cooling and the temperature of the strip at the beginning and end of the cooling process [1]. Since the strip is typically cooled from about $900^{\circ} \mathrm{C}$, to about $650^{\circ} \mathrm{C}$, the thermal conductivity and specific heat of the strip do not remain constant during the cooling process.

Yaniro K., Yamasaki J. et al. [2] have used the linear one dimensional heat conduction equation by considering the strip conduction in the thickness direction as a model for predicting the strip temperatures. But the model does not take into account the variation of thermal conductivity and specific heat of the strip with temperature. Although the authors have used the latent heat term in the model equation, the proposed analytical solution of the model equation does not take into account the latent heat term. Uetz G., Woelk G. et al. [3] have neglected the latent heat of phase transformation and approximated the model equation by an ordinary differential equation by assuming the temperature distribution along the thickness of the 
strip as a parabola. Yanagi K. [4] has modelled the process as an one dimensional heat conduction equation taking into account the latent heat of phase transformation but has neglected the variation of thermophysical properties of the strip with temperature. Ditzhuijzen G.V. [5] has used a model of the cooling process for the purposes of control, but the model neglects the conduction along the thickness of the strip, the latent heat of phase transformation and the variation of thermophysical properties of the strip with temperature. The model equation used in the work reported by Fillipovic J., Visakanta et al. [6] for offline calculations takes into account most of the factors that affect the temperature of the strip on the ROT but neglects the latent heat of phase transformation. Most of the other models reported in the literature are empirical in nature $[7,8,9]$.

The variation of thermal conductivity $\left(k_{u}\right)$ and specific heat $\left(c_{u}\right)$ with temperature are known and available in literature [10] for different steels. In the present work, it is proposed to take the variation of $k_{u}$ and $c_{u}$ into account by fitting polynomials from the available data. For the practical temperature ranges of the strip on the ROT, $k_{u}$ and $c_{u}$ are found to sary with temperature as per the following equations.

$$
\begin{aligned}
& k_{u}=a_{1}-b_{1} u \mathrm{~W} / \mathrm{m}^{\circ} \mathrm{C} \\
& c_{u}=383.7667+0.7069 u \text { Joules } / \mathrm{Kg}{ }^{\circ} \mathrm{C}
\end{aligned}
$$

where $a_{1}, b_{1}$ are coefficients which depend on the grade of steel. The values of $a_{1}$ and $b_{1}$ for two typical grades of steel are given below:

Carbon steel: $a_{1}=61.8474, b_{1}=0.0437$

Carbon-Silicon Steel: $a_{1}=53.7294, b_{1}=0.0329$

In the present work, the cooling process on the ROT has been modelled as an one dimensional heat conduction equation [11]:

$$
\rho c_{u} \frac{\partial u}{\partial t}=\frac{\partial\left(k_{u}(\partial u / \partial y)\right)}{\partial y}+H_{u} \gamma_{u}
$$

The boundary conditions when the strip is under a water zone are:

$$
\partial u / \partial y=\left(h_{a} / k_{u}\right)\left(u-u_{w}\right) \text { at } \mathrm{y}=0
$$

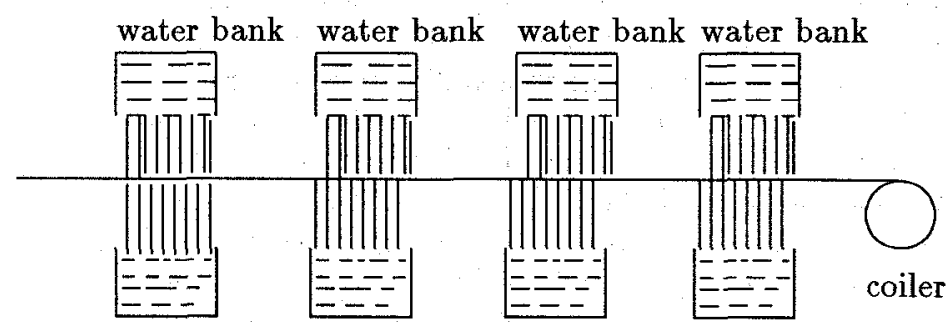

water bank water bank water bank water bank

$$
\partial u / \partial y=-\left(h_{b} / k_{u}\right)\left(u-u_{w}\right) \text { at } \mathbf{y}=\mathbf{s}
$$

When the strip is under an air zone the boundary conditions are [12]:

$$
\begin{aligned}
& \partial u / \partial y=\left(h_{a i r} / k_{u}\right)\left(u-u_{a i r}\right) \text { at } \mathrm{y}=0 \\
& \partial u / \partial y=-\left(h_{a i r} / k_{u}\right)\left(u-u_{a i r}\right) \text { at } \mathrm{y}=\mathrm{s}
\end{aligned}
$$

The initial condition is $\mathrm{u}=u_{s t}$ at $\mathrm{t}=0$ over the entire strip.

$$
\begin{aligned}
& \mathrm{t}=\text { time } \\
& \mathrm{y}=\text { co-ordinate in the thickness direction } \\
& \mathrm{u}=\text { temperature of strip } \\
& u_{w}=\text { temperature of water } \\
& \mathrm{s}=\text { thickness of the strip } \\
& \rho=\text { density of the strip } \\
& H_{u}=\text { latent heat of phase transformation } \\
& c_{u}=\text { specific heat } \\
& k_{u}=\text { thermal conductivity } \\
& h_{a}, h_{b}=\text { heat transfer co-efficients in the water zone } \\
& h_{\text {air }}=\sigma \epsilon\left(u^{2}-u_{\text {air }}^{2}\right)\left(u+u_{\text {air }}\right) \\
& \sigma=\text { Stefan-Boltzmann constant } \\
& \quad=5.67 \times 10^{-8} W / m^{2} K^{4} \\
& \epsilon=\text { emmisivity of steel }=0.8 \\
& u_{a i r}=\text { temperature of atmosphere } \approx u_{w} \\
& \gamma_{u}=\text { rate of phase transformation }
\end{aligned}
$$

Equation 1, henceforth referred to as the model equation, is a non-linear partial differential equation with nonlinear boundary conditions. Hence, a numerical method must be used to solve the model equation.

\section{Solution of the Model Equation}

Employing a numerical method for the solution of the model equation would require discretisation of the equation both in time and space. In order to have uniform step sizes for strips of different thickness and temperature, equation 1 has been normalised with respect to temperature and thickness by defining $T=\left(u-u_{w}\right) /\left(u_{i n i}-u_{w}\right)$ and $x=y / s$. The model equation with the above normalisation becomes

$$
s^{2} \rho \mathbf{c}_{T} \frac{\partial T}{\partial t}=\frac{\partial\left(k_{T}(\partial T / \partial x)\right)}{\partial x}+s^{2} H_{T} \gamma_{T}
$$

with the boundary conditions:

$$
\begin{aligned}
& \partial T / \partial x=\left(h_{1} / k_{T}\right) T \text { at } \mathrm{x}=0 \\
& \partial T / \partial x=-\left(h_{2} / k_{T}\right) T \text { at } \mathrm{x}=1 \\
& \text { with initial condition } \mathrm{T}=1 \text { at } \mathrm{t}=0 . \\
& h_{1}, h_{2}=\text { Heat transfer co-efficients as } \\
& \text { appropriate for the different zones. }
\end{aligned}
$$

The boundary condition of the strip suddenly changes from a Drichlet boundary condition to a Robbins boundary condition when the strip enters the ROT. Direct application of any of the numerical methods from $t=0$ will, 
therefore, result in unacceptable errors in the calculation of $\partial T / \partial x$ and consequently in the calculation of temperature. Thus it is necessary to use an analytical solution for the first time step even if it implies ignoring the variation of physical parameters with temperature and linearising equation 2 [11]. The phase transformation of steel begins only around $723^{\circ} \mathrm{C}$ [1] and no heat is liberated during the first time step when the temperature of the strip is typically around $900^{\circ} \mathrm{C}$. Hence, the following analytical solution for the linearised version of equation 2 has been used for the first time step [11] without the latent heat term.

$$
T=\sum_{n=1}^{\infty} Z_{n}(x) \exp \left(-k \beta_{n}^{2} t / \rho c\right) \int_{0}^{1} Z_{n}(v) d v
$$

where

$$
Z_{n}(x)=\frac{\left(\beta_{n} \cos \left(\beta_{n} x\right)+h_{1} \sin \left(\beta_{n} x\right)\right) \sqrt{2\left(\beta_{n}^{2}+h_{2}^{2}\right)}}{\sqrt{\left(\beta_{n}^{2}+h_{1}^{2}\right)\left(\beta_{n}^{2}+h_{2}^{2}+h_{2}\right)+h_{1}\left(\beta_{n}^{2}+h_{2}^{2}\right)}}
$$

and $\beta_{n}, \mathrm{n}=1,2, \ldots$ are the roots of

$$
\left(\beta^{2}-h_{1} h_{2}\right) \sin (\beta)=\beta\left(h_{1}+h_{2}\right) \cos (\beta)
$$

In order to compute the term $H_{T} \gamma_{T}$ in equation 2 the rate of phase transformation $\gamma_{T}$ has been calculated from Time Temperature Transformation (TTT) diagrams [1]. TTT diagrams give the amount of phase transformation that would take place with time for different strip temperatures when the material is held at constant temperature. In the present work, the points on the TTT curves [13] are stored as tables and the amount of phase transformation corresponding to a particular temperature and time is found by cubic spline interpolation [14] at each time step. The calculated transformation divided by the time step gives the rate of phase transformation $\gamma_{T}$. The value of latent heat of phase transformation $H_{T}$, has been calculated from the values of latent heats for the transformation of steel given by Hultgren $\mathrm{H}$. et al. [15].

The model equation is a partial differential equation (PDE) and the commonly used numerical methods for the solution of PDEs are the finite difference method, the boundary element method, the finite element method, the orthogonal collocation method and the integral profile method $[16,14,17,18,19,20,21]$. The boundary element method is well suited to elliptic type PDEs [22] whereas the model equation is a parabolic type of PDE. Further, the model equation is a one dimensional problem (in space) with a regular geometry and for such problems the finite element method and the orthogonal collocation method become essentially the same. The orthogonal collocation method has the additional advantage of requiring less number of terms to be computed when compared to the finite element method [20]. Hence in this work, the solution of the model equation has been attempted by the finite difference method, the orthogonal collocation method and the integral profile method only and the suitability of these three methods for solving the model equation has been examined from the point of view of accuracy of the solution and computational time.

For the purpose of ascertaining the accuracy of the numerical methods, the heat generation due to phase transformation was ignored as the contribution due to the phase transformation term in the model equation is independent of the numerical method used for the solution. In addition, equation 2 was linearised by taking the average values of $k_{u}$ and $c_{u}$ over the temperature range of the strip on the ROT resulting in equation 4.

$$
s^{2} c \rho \frac{\partial T}{\partial t}=\frac{\partial(k(\partial T / \partial x))}{\partial x}
$$

with the boundary conditions:

$\partial T / \partial x=\left(h_{1} / k\right) T$ at $\mathrm{x}=0$ and $\partial T / \partial x=-\left(h_{2} / k\right) T$ at $\mathrm{x}$ $=1$ and initial condition $\mathrm{T}=1$ at $\mathrm{t}=0$. where ' $\mathrm{k}$ ' and ' $c$ ' are the average values of thermal conductivity and specific heat respectively.

The accuracy of the three numerical methods was tested by comparing the solution of equation 4 obtained by the numerical methods and the analytical method (equation 3). Tests were carried out for strips of thickness ranging from $1 \mathrm{~mm}$ to $1 \mathrm{~cm}$ moving at velocities ranging from 3 meters/sec to 10 meters/sec (depending on strip thickness) on a 150 meters long ROT.

\subsection{Solution by Finite Difference Method (FDM)}

The finite difference formulation for the solution of the model equation has been carried out by the control volume approach [16]. In control volume form equation 2 for a finite volume can be written as

$$
s^{2} R \rho \mathrm{C}_{\bar{T}} \frac{\partial \bar{T}}{\partial t}=-\iint q n d S+s^{2} R H_{\bar{T}} \gamma_{\bar{T}}
$$

where

$q=$ heat flux vector

$\mathrm{R}=$ Volume of the region

$\mathrm{n}=$ unit outward normal to the surface

$\bar{T}=$ average value of $\mathrm{T}$ over the Control Volume

$\mathrm{dS}=$ Surface element

Consider a typical internal control volume such as the one labelled $\mathrm{A}$ in figure 2. $\bar{T}$ is taken as the value of $\mathrm{T}$ at the grid point (center of the control volume) and equation 5 is represented in the finite difference form. In the present work the implicit form of the finite difference formulation has been used but the physical parameters which vary with temperature have been evaluated explicitly. A complete explicit formulation was found to require time step of less 


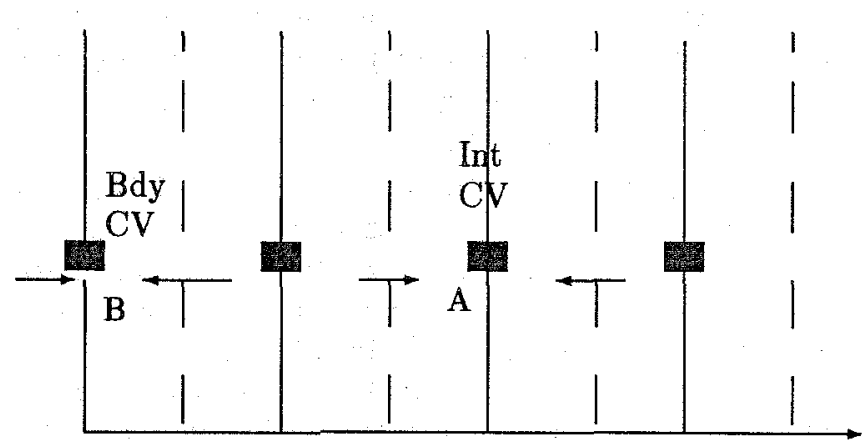

Bdy CV - Boundary Control Volume Int CV - Internal Control Volume

Figure 2: Control Volumes for the Problem

than 0.03 second for a stable solution even with grid spacing of $0.5 \mathrm{~mm}$ for a $5 \mathrm{~mm}$ thick strip, as calculated from stability constraints [16] and hence not used. Thus the difference representation of equation 5 with the proposed formulation is

$$
\begin{aligned}
& s^{2} \rho \mathrm{c}_{T} \frac{\left(T_{j}^{n+1}-T_{j}^{n}\right)}{\Delta t} \Delta x=\left.k_{T} \frac{\partial T}{\partial x}\right|_{j+1 / 2} ^{n+1}- \\
& \left.k_{T} \frac{\partial T}{\partial x}\right|_{j-1 / 2} ^{n+1}+ \\
& s^{2} H_{T} \gamma_{T}
\end{aligned}
$$

The subscript $\mathrm{j}$ represents a grid point and the superscript $\mathrm{n}$ represents the time step at which the quantity is evaluated.

Define $T_{*}=T_{j}^{n}, T_{+}=\left(T_{j}^{n}+T_{j+1}^{n}\right) / 2, T_{-}=\left(T_{j}^{n}+T_{j-1}^{n}\right) / 2$

$a_{1}=\frac{k_{T_{+}} \Delta t}{s^{2} \rho \mathrm{C}_{T_{*}}(\Delta x)^{2}}, a_{2}=\frac{k_{T_{-}} \Delta t}{s^{2} \rho \mathrm{C}_{T_{*}}(\Delta x)^{2}}, a_{3}=\frac{H_{T_{*}} \gamma_{T_{*}} \Delta t}{\rho \mathrm{C}_{T_{*}}}$

With the above definitions equation 6 for an interior grid point reduces to

$$
-a_{1} T_{j+1}^{n+1}+\left(1+a_{1}+a_{2}\right) T_{j}^{n+1}-a_{2} T_{j-1}^{n+1}=T_{j}^{n}+a_{3}
$$

For a boundary control volume the finite difference representation of equation 5 is obtained in a manner described above for the internal control volume except that the width of the control volume is $\Delta x / 2$. The term on the right hand side of equation 6 corresponding to either $(j+1 / 2)$ or $(j-1 / 2)$ is substituted with the boundary condition at $x=0$ or at $x=1$ as the case may be. The final form of the equations are:

$$
-2 a_{1} T_{j+1}^{n+1}+\left(1+2 a_{1}+a_{4}\right) T_{j}^{n+1}=T_{j}^{n}+\alpha_{3} ; \mathrm{x}=0
$$

$$
\left(1+2 a_{2}+a_{5}\right) T_{j}^{n+1}-2 a_{2} T_{j-1}^{n+1}=T_{j}^{n}+a_{3} ; \mathrm{x}=1
$$

where $a_{4}=\frac{2 h_{1} \Delta t}{s^{2} \rho \mathrm{C}_{T_{*}} \Delta x}, a_{5}=\frac{2 h_{2} \Delta t}{s^{2} \rho \mathrm{C}_{T_{*}} \Delta x}$

Equation 8 is applied to all the internal points and equations 9,10 are applied at the boundaries at each time step to get a tridiagonal system of linear equations of the form

$$
A x=b
$$

The tridiagonal system of linear equations (equation 11) are solved to get the new values of temperature for the current time step.

\subsubsection{Accuracy of FDM Solution}

In order to determine the largest time step and grid spacing that would give an accuracy of $1^{\circ}$ centigrade, the solution obtained by FDM for the linearised version of the model with constant heat transfer coefficients has been compared with that of the analytical solution. The step sizes $(\Delta t)$ and grid spacing $(\Delta x)$ were gradually decreased in steps of 0.1 starting from step sizes of 1 and a grid spacing of 0.5 till the desired accuracy of $1^{\circ}$ centigrade for the linear model was obtained. A time step of 0.1 second and a grid spacing of 0.1 has been found to give the desired accuracy.

In order to check the stability of the solution or the error propagation with time the following numerical experiment has been carried out. The errors $(\epsilon)$ between the FDM solution and the analytical solution for the linear model at the end of the second time step at each grid point was recorded. These errors $(\epsilon)$ were added to the FDM solution at the end of the second time step for the nonlinear model and the solution for the entire period of 15 seconds has been obtained. The difference between the solution obtained by FDM with and without the addition of errors has been recorded at each time step. This difference essentially gives the propagation of the errors $(\epsilon)$ introduced at the end of the second time step. The propagation of error with time is shown in figure 3 . Since the error decays with time the solution obtained by FDM is stable.

\subsubsection{Parallel Solution by FDM}

A parallel solution of the above FDM formulation has been implemented on a linear array of transputers [23]. The task of computation of $a_{1}, a_{2}, a_{3}, a_{4}$ and $a_{5}$ in equations 8 to 10 for the various grid points (elements of matrix $A$ in equation 11) at each time step has been evenly distributed among the transputers [24]. Let ' $P$ ' be the number of transputers in the network, ' $\mathrm{g}$ ' the number of grid points and ' $R$ ' the remainder when $g$ is divided by $P$. Then the first $\mathrm{R}$ transputers would calculate the elements for $(\lfloor g / p\rfloor+1)$ rows of $A$ and the remaining transputers would calculate for $\lfloor g / p\rfloor$ rows of A. The solution is propagated in time sequentially. 


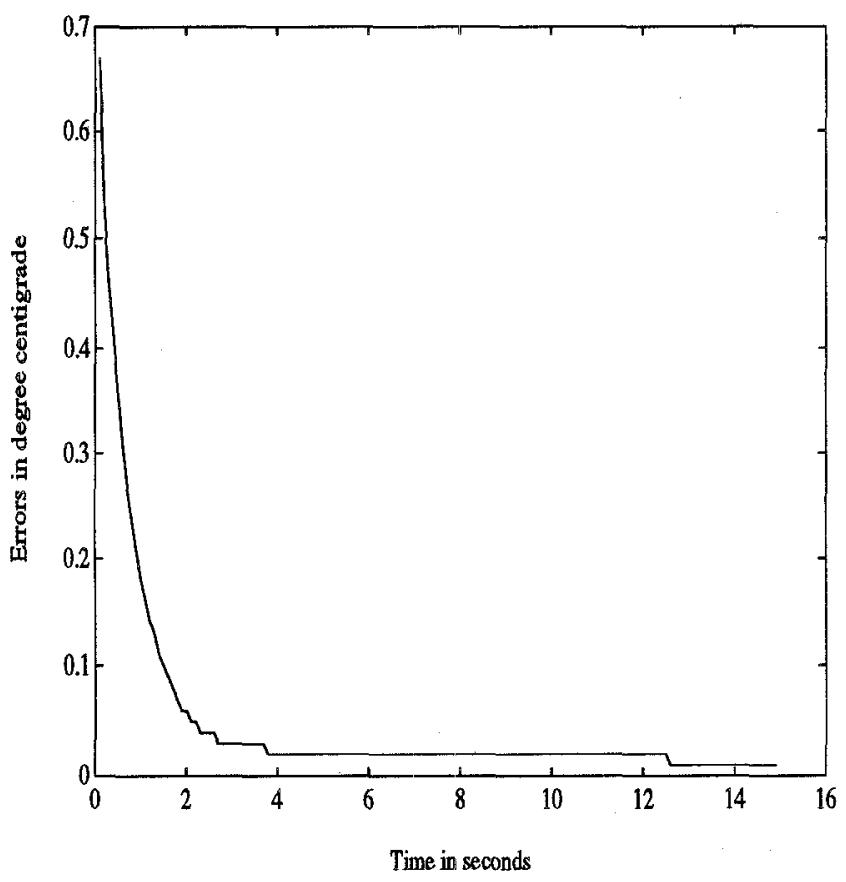

Figure 3: Error Propagation in FDM

For calculation of $T_{+}$and $T_{-}$(equation 7 ) for a grid point ' $i$ ' at the end of each time step, the values of temperatures for the previous time step at the adjacent grid points ((i-1) and $(i+1))$ are required. Hence each transputer communicates the temperature values of the first and last grid points assigned to each transputer to the adjacent transputers. Since the communication requirement is only between adjacent transputers a linear array is sufficient to communicate the values through a direct link between the communicating transputers.

The tridiagonal system of linear equations which results by using a grid spacing of 0.1 is a $11 \times 11$ system of linear equations. Parallel methods for solution of the tridiagonal system of equations are generally efficient only when the order of the tridiagonal system is 500 or higher while in the present case the tridiagonal system is of order $11 \times 11$. However, in order to verify whether any improvement can be achieved by solving the tridiagonal system(equation 11) in parallel, two representative methods for the parallel solution of equation 11, namelly the parallel prefix recursive doubling algorithm proposed by Egecioglu [25] and the parallel algorithm based on reducing the matrix $A$ to diagonal form by column sweep techniques proposed by Evans [26], have been attempted and the performance compared with the sequential solution of the tridiagonal system. While solving the tridiagonal system of equations by each of the three methods mentioned above, the coefficients of matrix A were computed in parallel. The time taken for the solution of the model equation by FDM with the co-efficients of matrix $A$ in equation 11 computed in parallel and the tridiagonal system being solved by the three methods with different number of transputers in the network are given in table 1.

As was expected parallel methods of solving tridiagonal system of linear equations takes more time when compared with the ordinary sequential method of solving the tridiagonal system. Table 1 also indicates that the speed-up saturates with four processors.

\subsection{Orthogonal Collocation Method}

In the orthogonal collocation method the unknown variable is assumed to be a linear combination of independent orthogonal polynomials [20]. In this work the unknown variable $\mathrm{T}$ in equation 2 has been taken as

$$
T=b+c x+x(1-x) \sum_{i=1}^{N} \alpha_{i} P_{i-1}(x)
$$

where $\mathrm{N}$ is the number of interior collocation points apart from the two boundary points, $P_{i-1}(x)$ is a polynomial of degree (i-1) and is orthogonal to all polynomials of degree less than (i-1). The $\mathrm{N}$ interior collocation points are taken as the roots of $P_{N}$ and the remaining two collocation points are taken as the boundary points. There are totally $(\mathrm{N}+2)$ coefficients to be determined in equation 12. The assumed profile for $T$ in equation 12 is substituted in equation 2 and the residual $R=s^{2} \rho \mathrm{c}_{T}(\partial T / \partial t)-\partial\left(k_{T}(\partial T / \partial x)\right) / \partial x-s^{2} H_{T} \gamma_{T}$ is set equal to zero at the $\mathrm{N}$ interior collocation points which gives $\mathrm{N}$ equations. The boundary conditions further give two more equations thus resulting in $(\mathrm{N}+2)$ equations to be solved for the $(\mathrm{N}+2)$ unknowns. Once the co-efficients $\mathrm{b}, \mathrm{c}, \alpha_{i}$ are determined the temperature $\mathrm{T}$ at any point can be found from equation 12 .

From the point of view of implementation the computer programs are simpler if they are written in terms of the unknown values at the collocation points rather than in terms of co-efficients [20]. Since the trial function for the unknown in equation 12 is polynomial of degree $(N+1)$ in $x$, the trial function can be written as

$$
T\left(x_{j}\right)=\sum_{i=1}^{N+2} x_{j}^{i-1} d_{i}
$$

where $x_{j}$ is a collocation point. Taking the first derivative and the Laplacian of equation 13 at the collocation points:

$$
\begin{array}{r}
\left.\frac{d T}{d x}\right|_{x_{j}}=\left.\sum_{i=1}^{N+2} \frac{d x^{i-1}}{d x}\right|_{x_{j}} d_{i} \\
\left.\nabla^{2}(T)\right|_{x_{j}}=\left.\sum_{i=1}^{N+2} \nabla^{2}\left(x^{i-1}\right)\right|_{x_{j}} d_{i}
\end{array}
$$


Table 1: Solution Time with FDM

\begin{tabular}{|c|c|c|c|}
\hline $\begin{array}{c}\text { Method Used } \\
\text { for Tridiagonal } \\
\text { System Solution }\end{array}$ & $\begin{array}{c}\text { Sequential } \\
\text { Method }\end{array}$ & $\begin{array}{c}\text { Parallel Column } \\
\text { Sweep Method }\end{array}$ & $\begin{array}{c}\text { Parallel Prefix } \\
\text { Method }\end{array}$ \\
\hline $\begin{array}{c}\text { Number of } \\
\text { Processors }\end{array}$ & $\begin{array}{c}\text { Time } \\
\text { milli seconds }\end{array}$ & $\begin{array}{c}\text { Time } \\
\text { milli seconds }\end{array}$ & $\begin{array}{c}\text { Time } \\
\text { milli seconds }\end{array}$ \\
\hline 1 & 540.9 & 619.6 & 601.9 \\
\hline 2 & 326.3 & 388.2 & 406.8 \\
\hline 3 & 253.8 & 314.1 & 349.2 \\
\hline 4 & 222.6 & 280.4 & 298.4 \\
\hline 5 & 231.7 & 284.8 & 316.4 \\
\hline
\end{tabular}

Writing the above equations for all the collocation points, equations 13 to 15 can be written in matrix notation as follows:

$$
\begin{gathered}
{[T]=[Q][d]} \\
{\left[\frac{d T}{d x}\right]=[C][d],\left[\nabla^{2} T\right]=[D][d]}
\end{gathered}
$$

where $Q_{j i}=x_{j}^{i-1}, C_{j i}=(i-1) x_{j}^{i-2}$ and $D_{j i}=(i-1)(i-$ $2) x_{j}^{i-3}$. Substituting for [d] obtained from equation 16 , equations 17 can be written as

$$
\begin{gathered}
{\left[\frac{d T}{d x}\right]=[c]\left[Q^{-1}\right][T] \equiv[A][T] \text { and }} \\
{\left[\nabla^{2} T\right]=[D]\left[Q^{-1}\right][T] \equiv[B][T] .}
\end{gathered}
$$

In order to formulate the solution for equation 2 by the orthogonal collocation method, equation 2 is written as

$$
s^{2} \rho \mathrm{c}_{T} \frac{\partial T}{\partial t}=k_{T} \frac{\partial^{2} T}{\partial x^{2}}+\frac{d k_{T}}{d T}\left(\frac{\partial T}{\partial x}\right)^{2}+s^{2} H_{T} \gamma_{T}
$$

Since $k_{T}$ is of the form $\beta+\delta T, d k_{T} / d T=\delta$, the above equation can be written as

$$
s^{2} \rho \mathrm{C}_{T} \frac{\partial T}{\partial t}=k_{T} \frac{\partial^{2} T}{\partial x^{2}}+\delta\left(\frac{\partial T}{\partial x}\right)^{2}+s^{2} H_{T} \gamma_{T}
$$

with the boundary conditions:

$$
\frac{\partial T}{\partial x}=\frac{h_{1}}{k_{T}} T \text { at } x=0 \text { and } \frac{\partial T}{\partial x}=-\frac{h_{2}}{k_{T}} T \text { at } x=1
$$

and initial condition $\mathrm{T}=1$.

Substituting for $\mathrm{T}, \partial^{2} T / \partial x^{2}$ and $\partial T / \partial x$ in equations 19 and 20 from equations 16 and 18 , the final set of equations for the solution of equation 2 by the orthogonal collocation method is obtained as follows:

$$
\text { For } \mathrm{j}=1,2,3, \ldots, \mathrm{N}
$$

$$
\begin{aligned}
\left.s^{2} \rho \mathbf{c}_{T} \frac{\partial T}{\partial t}\right|_{x_{j}}= & k_{T} \sum_{i=1}^{N+2} B_{j i} T_{i}+\delta\left(\sum_{i=1}^{N+2} A_{j} i T_{i}\right)^{2}+ \\
& s^{2} H_{T} \gamma_{T} \\
\sum_{i=1}^{N+2} A_{j i} T_{i}= & \left(s h_{1} / k_{T}\right) T_{j}, j=1 \\
\sum_{i=1}^{N+2} A_{j i} T_{i}= & -\left(s h_{1} / k_{T}\right) T_{j}, j=N+2
\end{aligned}
$$

Equation 21 is a set of $\mathrm{N}$ simultaneous ODEs and equations 22 and 23 are linear equations.

\subsubsection{Implementation and Accuracy of Orthogo- nal Collocation Method}

In order to determine the accuracy of the solution equations 21 was solved by the fourth order Runge Kutta method [14] and equations 22 and 23 were solved explicitly at each time step. The largest time step and the least number of collocation points that would yield an accuracy of $1^{\circ}$ centigrade has been determined in a manner similar to the one employed for the FDM method. It has been found that a time step of 0.1 second and 6 collocation points yields the desired accuracy. When, the number of collocation points were increased beyond 6 , the ODEs became stiff requiring a smaller time step for a stable solution. The model equation solution by the orthogonal collocation method has been compared with solution obtained by the FDM method and it has been found that the difference between the FDM solution and orthogonal collocation solution is of the order of $2^{\circ}$ centigrade. The propagation of error with time has also been studied for 6 collocation points as has been explained for the FDM method. The error propagation with time is plotted in figure 4. As can be seen from the figure the error decays with time indicating that the solution is stable. Although the Runge Kutta method yields a stable solution, the method is computationally intensive and is not as amenable to parallelisation as the block predictor corrector methods. Hence, the solution of equations 21 has also been attempted by two other 


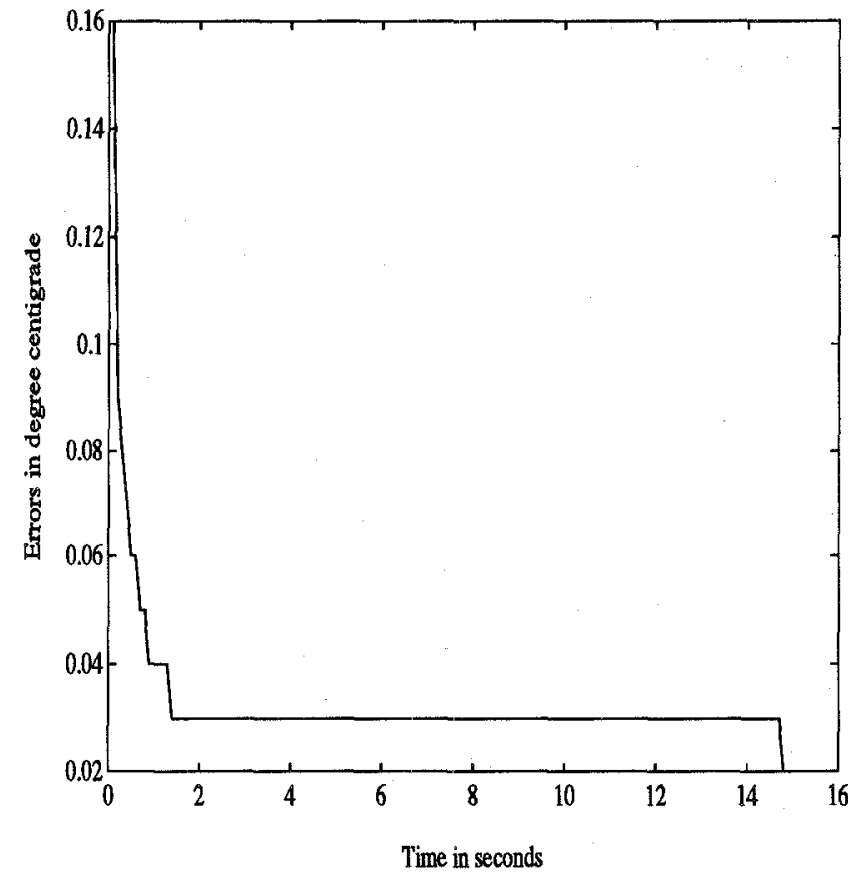

Figure 4: Error Propagation in Orthogonal Collocation

methods, namely, the modified midpoint method and a block predictor corrector method proposed by Shampine and Watts [27]. The modified midpoint method has been tried since the method is computationally less intensive than the Runge Kutta method. The block predictor corrector method is more amenable to parallelisation than the Runge Kutta method because in each predictor step the predictor values for two subsequent time steps can be computed in parallel. Similarly in each corrector step the corrector values for two subsequent time steps can be computed in parallel. However, both these methods turned out to be unstable for the solution of equations 21 and have not been considered further.

\subsubsection{Parallel Solution}

The solution of equations 21 to 23 have been implemented in parallel by evenly distributing the solution of the $(\mathrm{N}+2)$ equations at each time step among the processors and propogating the solution in time sequentially. Since the Runge Kutta method has been used for the solution of ODEs, solution of ODEs at each time step requires four substeps. After each Runge Kutta substep, the temperatures at all the collocation points as calculated by the Runge Kutta method are required for the calculation of the value of temperature at each collocation point in the subsequent substep. Thus each processor ' $P$ ' has to communicate the values of the temperatures at the collocation points calculated in ' $\mathrm{P}$ ' to all the other processors in the network at the end of every Runge Kutta substep.
The parallel solution has been implemented on a multitransputer system with upto 3 transputers in the network. Since every transputer requires to communicate with every other transputer in the network, the transputers were connected as a ring so that there is a direct link between any two transputers. The time taken for solution with 13 transputers in the network are shown in table 2. Due to the excessive communication requirement, the speed-up saturates with the number of processors equal to 3 itself.

Table 2: Time taken with Orthogonal Collocation

\begin{tabular}{|c|c|}
\hline $\begin{array}{c}\text { Number of } \\
\text { Processors }\end{array}$ & $\begin{array}{c}\text { Time in } \\
\text { milli seconds }\end{array}$ \\
\hline 1 & 739.1 \\
\hline 2 & 408.5 \\
\hline 3 & 403.0 \\
\hline
\end{tabular}

A comparison of tables 1 and 2 clearly shows that FDM takes less time when compared to orthogonal collocation method.

\subsection{Integral Profile Method (ITPF)}

In contrast to FDM and orthogonal collocation, the integral profile method satisfies the PDE in an integral sense and not pointwise. The dependent variable is assumed to be a polynomial and the coefficients of the polynomial are determined by the boundary conditions and by satisfying the PDE in an integral sense $[20,21]$. Let

$$
T(x, t)=a+b x+c x^{2}
$$

For a symmetrical case $\left(h_{1}=h_{2}=h\right.$ in equation 2), applying the boundary conditions of the problem yield

$$
T(x, t)=T(0, t)+\frac{h}{k_{T}} T(0, t) x-\frac{h}{k_{T}} T(0, t) x^{2}
$$

In order to find $T(0, t)$, equation 25 is substituted in equation 2 . The resulting equation is integrated over the entire domain from $x=0$ to $x=1$ to satisfy the PDE in an integral sense to get

$$
\begin{aligned}
& s^{2} \rho \int_{0}^{1} \mathrm{c}_{T} \frac{\partial}{\partial t}\left(T(0, t)+\frac{h}{k_{T}} T(0, t) x-\frac{h}{k_{T}} T(0, t) x^{2}\right)= \\
& \left.k_{T} \frac{\partial T}{\partial x}\right|_{x=1}-\left.k_{T} \frac{\partial T}{\partial x}\right|_{x=0}+s^{2} H_{\bar{T}} \gamma_{\bar{T}}(26)
\end{aligned}
$$

where $\bar{T}$ represents a average value. Taking $\mathrm{C}_{T}=(\alpha+\beta T)$, $k_{T}=\delta+\sigma T$ and substituting the boundary conditions in equation 26 , the following ordinary differential equation is obtained.

$$
\frac{\partial \tau}{\partial t}=\frac{-2 h \mathcal{T}+s^{2} H_{\bar{T}} \gamma_{\bar{T}}}{30 k_{\tau}^{3}(U+V)}
$$

where 


$$
\begin{aligned}
& \tau=T(0, t) \\
& U=s^{2} \rho \alpha k_{\tau}\left[k_{\tau}\left(30 k_{\tau}+5 h\right)-5 h \sigma \tau\right] \\
& V=s^{2} \rho \beta \tau\left[k_{\tau}\left\{60 k_{\tau}^{2}+h\left(20 k_{\tau}-10 \sigma \tau+2 h\right)\right\}-2 h^{2} \sigma \tau\right]
\end{aligned}
$$

Equation 27 is solved to get the value of $\mathrm{T}(0, \mathrm{t})$ which can then be substituted in equation 25 to get the values of $\mathrm{T}$ at various points in the domain at each time step.

\subsubsection{Accuracy of the ITPF method}

The integral profile method has been implemented for the symmetric case $\left(h_{1}=h_{2}=h\right)$ and the ODE has been solved by the fourth order Runge Kutta method. The solution by the integral profile method has been compared with the solution obtained by FDM. Although the solution by the integral profile method has been found to be stable, the errors increased with strip thickness from about $2^{\circ}$ centigrade for $2 \mathrm{~mm}$ thick strip, to $15^{\circ}$ for a $1 \mathrm{~cm}$ thick strip on an average. Such large errors in the solution are not acceptable and hence the integral profile method has not been pursued further.

\section{Conclusions}

The cooling process on the runout table of a hot strip has been modelled by taking into account the various factors that affect the temperature of the strip on the ROT such as convection to the sprayed water, radiation to the atmosphere, conduction along the thickness direction, latent heat of phase transformation and the variation of thermal conductivity and specific heat of the strip wtih temperature. The cooling process has been modelled as an one dimensional non linear heat conduction equation with convection and radiation boundary conditions.

The formulation and feasibility of the solution of the model equation by three numerical methods namely the finite difference method, the orthogonal collocation method and the integral profile method have been studied. Of the three methods, the orthogonal collocation method and FDM were found to give accurate solutions and these two methods were implemented on a multiprocessor system with transputers as the processing elements. A parallel solution by the finite difference method on a linear array of four processors provided the best results in terms of accuracy, computation time and speed-up.

Based on the studies and results reported in this paper, a real time simulator has been proposed and developed for the ROT of hot strip mills which is the subject of a companion paper titled, "Real Time Simulator for the Runout Table of Hot Strip Mills".

\section{References}

[1] R.E. Reed. Physical Metallurgical Principles. Affiliated East-West Press PVT Ltd, New Delhi, 1964.
[2] K. Yaniro, J. Yamasaki, M. Furukawa, et al. Develoment of Coiling Temperature Control System on Hot Strip Mill. Technical Report No. 24, Kawasaki Steel, Apr 1991.

[3] G. Uetz, G. Woelk, and T. Bischops. Influencing the formation of the steel structure by suitable temperature control in the runout sections of hot strip mills. Steel Research, Vol. 62(No. 5):pp 216-222, May 1991.

[4] K. Yanagi. Prediction of strip temperature for hot strip mills. Transactions of the Iron ans Steel Institute of Japan, Vol. 16(No. 1):pp 11-19, Jan 1976.

[5] G.V. Ditzhuijzen. The controlled cooling of hot rolled strip: a combination of physical modelling, control problems and practical adaptation. IEEE Trans. on Automatic Control, Vol. 38(No. 7):pp 1060-1065, July 1993.

[6] J. Filipovic, R. Viskanta, F.P. Incropera, and T.A. Veslocki. Thermal behaviour of a moving steel strip cooled by an array of planar water jets. Steel Research, Vol. 63(No. 10):pp 438-446, Oct 1992.

[7] A.G. Groch, R. Gubernat, and E.R. Birstein. Automatic control of laminar flow cooling in continuous an reversing hot strip mills. Iron and Steel Engineer, Vol. 67(No. 9):pp 16-20, Sep 1990.

[8] M.D. Leltholf and J.R. Dahm. Model reference control of runout table cooling at LTV. Iron and Steel Engineer, Vol. 66(No. 8):pp 31-35, Aug 1989.

[9] R.W. Moffat, M.C. Moore, M.J. Robinson, and J.D. Ashton. Computer control of hot strip cooling temperature with variable flow laminar sprays. Iron and Steel Engineer, Vol. 62(No. 11):pp 21-28, Nov 1985.

[10] B.P. Bardes, editor. Metals Handbook. Volume Vol. 1, American Society for Metals, Ohio, 1978.

[11] H.S. Carslaw and J.C. Jaeger. Conduction of Heat in Solids. Oxford University Press, London, 1986.

[12] L.C Thomas. Fundamentals of Heat Transfer. Prentice-Hall, INC., New Jersey, 1980.

[13] Atlas of Isothermal and Cooling Transformation Diagrams. American Society for Metals, Ohio, 1977.

[14] W.H. Press, S.A. Teukolsky, W.T. Vetterling, and B.P. Flannery. Numerical Recipes in C. Cambridge University Press, CAMBRIDGE, 1993.

[15] H. Hultgren, P.D. Desai, D.T. Hawkin, et al., editors. Selected Values of Thermodynamic Properties of Binary Alloys. American Society of Metals, Ohio, 1973. 
[16] W.J. Minkowycz, E.M. Sparrow, G.E. Schneider, and R.H. Pletcher. Handbook of Numerical Heat Transfer. John Wiley \& Sons, Inc., 1988.

[17] C.A. Brebbia and S. Walker. Boundary Element Techniques in Engineering. Butterworth \& Co (Publishers) Ltd, London, 1980.

[18] A.J. Nowak. Temperature Fields in Domains with Heat Sources using Boundary only Formulation. In C.A. Brebbia, editor, Boundary Elements - X, pages pp 233-246, Springer Verlag, NEW YORK, 1988.

[19] C.S. Desai and J.F. Abel. Introduction to the Finite Element Method. Affliated East-West Press Ltd, New Delhi, 1972.

[20] B.A. Finlayson. The Method of Weighted Residuals and Variational Principles with Application in Fluid Mechanics, Heat and Mass Transfer. Academic Press, New York, 1972.

[21] T.R. Goodman. Application of Integral Methods to Transient Nonlinear Heat Transfer. In Jr. Thomas F. Irvine and James P. Hartnett, editors, $A d$ vances in Heat Transfer, pages pp 52-120, Academic press, NEW YORK, 1964.

[22] G.S. Gipson. Progress in the Analysis of Poisson Type Problems by Boundary Elements. In C.A. Brebbia, editor, Boundary Elements - X, pages pp 101-113, Springer Verlag, NEW YORK, 1988.

[23] R. Krishna Kumar, S.K. Sinha, and L.M. Patnaik. A fault-tolerant multi-transputer architecture. Microprocessors and Microsystems, Vol. 17(No. 2):pp 7581, Mar 1993.

[24] R. Krishna Kumar. A Fault-Tolerant Multitransputer Based Firmware for Parallel Solution of Partial Differential Equations by the Boundary Element Method. Master's thesis, Indian Institute of Science, Nov 1991.

[25] O. Egecioglu, C.K. Koc, and A.J. Laub. A Recursive Doubling Algorithm for Solution of Tridiagonal Systems on Hypercube Multiprocessors. Journal of Computational and Applied Mathematics, Vol. 27:pp 95-108, 1989.

[26] D.J. Evans. New Parallel Algorithms for PDEs. In Proceedings of the International Conference on Parallel Computing, pages pp 3-56, 1983.

[27] M.A. Franklin. Parallel solution of ordinary differential equations. IEEE Trans. on Computers, Vol. C-27(No. 5):pp 413-420, May 1978. 